\title{
Cooperative Strategies for Maximum-Flow Problem in Uncertain Decentralized Systems Using Reliability Analysis
}

\author{
Hadi Heidari Gharehbolagh, ${ }^{1}$ Ashkan Hafezalkotob, ${ }^{1}$ Ahmad Makui, ${ }^{2}$ and Sadigh Raissi ${ }^{1}$ \\ ${ }^{1}$ School of Industrial Engineering, Islamic Azad University, South Tehran Branch, Tehran 11518-63411, Iran \\ ${ }^{2}$ Department of Industrial Engineering, Iran University of Science and Technology, Tehran 16846-13114, Iran \\ Correspondence should be addressed to Ashkan Hafezalkotob; hafezalkotob@iust.ac.ir
}

Received 22 May 2016; Accepted 25 July 2016

Academic Editor: Arturo Pagano

Copyright (c) 2016 Hadi Heidari Gharehbolagh et al. This is an open access article distributed under the Creative Commons Attribution License, which permits unrestricted use, distribution, and reproduction in any medium, provided the original work is properly cited.

\begin{abstract}
This study investigates a multiowner maximum-flow network problem, which suffers from risky events. Uncertain conditions effect on proper estimation and ignoring them may mislead decision makers by overestimation. A key question is how self-governing owners in the network can cooperate with each other to maintain a reliable flow. Hence, the question is answered by providing a mathematical programming model based on applying the triangular reliability function in the decentralized networks. The proposed method concentrates on multiowner networks which suffer from risky time, cost, and capacity parameters for each network's arcs. Some cooperative game methods such as $\tau$-value, Shapley, and core center are presented to fairly distribute extra profit of cooperation. A numerical example including sensitivity analysis and the results of comparisons are presented. Indeed, the proposed method provides more reality in decision-making for risky systems, hence leading to significant profits in terms of real cost estimation when compared with unforeseen effects.
\end{abstract}

\section{Introduction}

Continuous development of technology in petrochemical industries, automobile manufacturing, water distribution networks, electricity industries, and transportation networks has created complex, competitive, and decentralized environments for suppliers [1]. This makes distribution companies and transport networks consider their main variables such as capacity, time, and cost as important elements in today's competitive environments and try to increase their service levels for customers and promote the products according to customers' demand [2]. Therefore, designing an appropriate system in decentralized networks with the consideration of uncertainty is one of the most important issues in today's competitive world.

In the design of suitable systems in decentralized networks, quality survival and reliability in decision-makings with nondeterministic circumstances are highly important. Since any failure in the network affects noticeably customer services and causes heavy costs to suppliers, increasing network reliability and network performance is extremely essential. One of the key tools to improve network performance in decentralized networks is game theory and cooperative games that can create optimal strategies for suppliers [3].

In uncertain circumstances, we have focused on real values of decision parameters such as time, cost, and capacity which may deploy from uncertain patterns. Such uncertain circumstances may arise due to various sources of fluctuations in supply/demand patterns, politics, traffic, natural disasters, war, falling debris, dropping voltage, and so on.

Naturally the effects of such unforeseen events may mislead decision makers. Accordingly, uncertainty decisionmaking is an important issue in today's competitive world. In this regard through this research, a novel mathematical programming model based on triangular probability distribution in nondeterministic decentralized systems is proposed to solve maximum-flow problem. Applying reliability function for triangular density function helped us to estimate a more reliable value for uncertain parameters of traveling time, associated transportation cost, and the actual amount of displacement capacity according to experts' subjective comments. 
The reminder of the paper is organized as follows: Literature review and research gap in decentralized networks are presented in Section 2. Through Section 3, the proposed mathematical model is presented. The properties of the constructed model and further cooperative investigation are evaluated through a numerical example in Section 4. Finally concluding remarks are presented in the last section.

\section{Literature Review}

The main related issues to the maximum-flow problems such as logistic network, cooperative game theory, and reliability have been discussed and the research gap is given as follows.

2.1. Logistic Network. Logistic network model is one of the most important models in mathematical programming and operations research and includes network planning and inventory control, production planning, planning and project control, facilities location, and many other applications. Moreover, it can be used in airlines, railways, roads, pipelines, and so forth. Besides, logistic network is one of the main issues in the maximum-flow problems [4]. Frisk et al. [5] worked on the collaboration between logistic companies in the forest industries by investigating a number of sharing mechanisms including nucleolus, Shapley value, shadow prices, separable and nonseparable costs, and volume weights. Lehoux et al. [6] considered different cooperation techniques such as the Shapley value, shadow prices, and nucleolus in logistic networks.

The purpose of the maximum-flow problem in the network is to reach the highest amount of transportation flow from the initial node to the terminal node by considering the capacity of the arcs.

2.2. Cooperative Game Theory (CGT). In the last decades, the logistic costs in distribution networks have been increased dramatically due to a noticeable raise in customers' expectations. To reduce the costs, various game theory methods and horizontal cooperative games are used in the logistic networks in which the horizontal cooperative games have led to getting higher payoff because of cooperation between companies [7].

Vanovermeire and Sörensen [8] used the cooperation techniques including the nucleolus and the Shapley value methods among shippers to increase their performance. The results indicated that the cooperation reduced the costs of distribution and delivery but the reduction is depending on the flexibility of the companies for delivery of goods.

Saha et al. [9] used two price promotion policies, MIR and RS, and DDD as tools in a manufacturer-distributerretailer channel to achieve improved individual profits and to eliminate channel conflict. Generalized Nash bargaining product could determine particular profit split in cooperative environment although it needs negotiation powers of all chain members.

Networks are often controlled by multiple owners. As a case in point, gas pipeline, which is an international system, has established an integrated network in Europe. In this case, each country controls some parts of the distribution network and in fact a cooperative game in the network is built $[5,10]$.

Charles and Hansen [11] proposed a theoretical cost saving mechanism for cost saving assignment in an enterprise network and global cost minimization by the help of CGT. The results showed that the cost allocations obtained through the activity based costing technique were stable and rational.

Zibaei et al. [12] proposed a multidepot vehicle routing problem for minimizing the transportation costs when there are multiple owners. The results indicate that the transportation costs were declined that could lead to noticeable cost savings. Therefore, several methods based on the CGT theory including $s$ value, Shapley value, least core, and equal cost saving method were proposed for a fair allocation of the cost savings between the owners.

Zhao et al. [13] recognized the game theory as a comprehensive tool for studying strategies of supply chain elements. Bell [14] optimized a transportation model from origin to destination with five considered paths and four scenarios by zero-sum cooperative games and using linear programming approach in order to minimize paths' costs. In the competitive market of emerging economies such as China and India, too much pressure on the global supply chain has caused new limitations for the countries' transportation networks. Reyes [15] used Shapley method in cooperative games for optimizing the transport network in order to stabilize the supply chain and reduce the excessive pressure. San Cristóba [16] optimized cost allocation between activities of networks by using game theory. Lozano et al. [7] introduced a mathematical programming model to measure the benefits of merging the transportation demands from different companies. Hafezalkotob and Makui [17] introduced a stochastic mathematical programming model for a multiple-owner graph problem. Their model was based on the cooperative game theory in order to indicate that the collaboration between independent owners of a logistic network will lead to maintaining a reliable maximum flow. McCain [18] concentrated on the cooperative games in collaborating organizations in order to analyse the effects of these games on the organization expansion and its profit. Esmaeili et al. [19] discussed guarantee services with game theory approach in three different levels: manufacturers, distributors, and customers. Guarantee is a service contract between manufacturers and customers and plays a key role in most of lawful business processes. Interaction between the players (manufacturers, distributors, and customers) is studied by noncooperative and semicooperative games to obtain optimum results including selling price, period's guarantee, guarantee's fee for manufacturers, costs of maintenance, and repair cost for distributors.

2.3. Reliability. One of the most important aspects of reliability is network reliability. The network reliability is defined as a capability or probability that a network system has to completely fulfill customer-tailored communications tasks during the stipulated successive operation procedure [20].

In the last decade, the reliability of the transport network and power distribution systems had been widely considered. The experience of incidents such as the Kobe earthquake, which occurred in Japan in 1995, made many researchers to 
identify and improve the reliability of the transport networks. Also, reliability is highly important in special cases such as poor weather conditions, disasters, road accidents, and terrorist attacks. Moreover, the increased economic activities all over the world have increased the importance of network systems and value of the network [21].

Zhao et al. [22] proposed stochastic simulation methods based on Monte Carlo by considering the system reliability and component probabilistic importance of a road network. Then a new system that incorporates this proposed method is developed. The system is a useful practical quantitative analysis tool to assist the decision-making for the road management departments, such as predicting the increased system reliability of a road network when it adds a new link, finding the key components that need to be upgraded or improved, and evaluating the system reliability of different road network planning schemes.

Hosseini and Wadbro [23] studied the essential problems of reliability and stability analysis in uncertain networks. They used uncertainty theory to make sure the arrival of relief materials and rescue vehicles to the disaster areas is in time. They then defined the new problems of $\alpha$-most reliable path ( $\alpha$-MRP), which aims to minimize the pessimistic risk value of a path under a given confidence level $\alpha$ and very most reliable path (VMRP) in an uncertain traffic network. Amin et al. [24] introduced an approach to software reliability prediction based on time series modeling in order to show the importance of reliability for quality systems. Therefore, with increasing attention to the quality, finding a way to enhance product's reliability is considered widely, because, to stay in competition environments, product quality and the associated costs have very important roles [25]. Many real-world systems such as power transmission systems can be a multicast flow network in which each independent part can have its own payoff and profit. Yeh et al. [26] calculated the reliability of networks in which the flow is run by multiplayers by using a new cut-based algorithm. KhaliliDamghani et al. [27] optimized the maximum reliability in the series-parallel systems by minimizing the weight and the value of the network with metaheuristic algorithm PSO. Hausken [28] measured the probability of the risk of reliability in series, parallel, and combined networks by using game theory. Szeto [21] used cooperative game to measure the reliability of conflicting reports on transport networks in order to minimize the cost of paths. Stackelberg and Nash equilibrium methods were used in optimization. The results of the research indicated that noncooperative games can lead to worse situations. Prabhu Gaonkar et al. [29] employed fuzzy models to study reliability in transportation network and Jiang et al. [30] assumed uncertain capacities in a transportation network design problem. Zhang et al. [31] dealt with a network resilience problem through studying topology in transportation networks.

2.4. Research Gap. To the best of the authors' knowledge, no study has been done on the cooperative games among different players in decentralized systems in nondeterministic circumstances with considering reliability. There is main contribution in this study with regard to a mathematical model

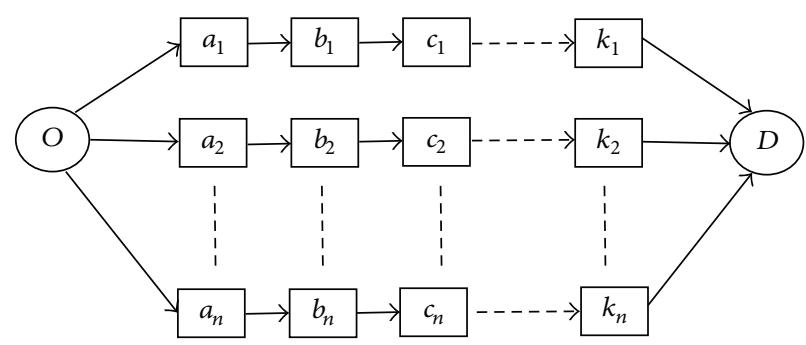

FIGURE 1: A typical network which is controlled by $n$ players.

based on triangular probability distribution in decentralized systems in nondeterministic circumstances for coalitions of owners/players. We have studied how cooperation among the multiple owners and the changes of flow parameters cost and time in nondeterministic circumstances can increase players' payoff and the amount of reliability in the network. Cooperation value also is measured by effectiveness (synergy) index. To address the problem of allocating the cooperation value to the cooperating owners, we have considered several methods of cooperative game theory.

\section{Material and Methods}

3.1. The Proposed Mathematical Model. The main framework of the considered problem is shown in Figure 1. Suppose that an interconnected decentralized system is controlled by $n$ players at the same time where $a_{1}, b_{1}, c_{1}, \ldots, k_{1}$ nodes are controlled by the 1st player and $a_{2}, b_{2}, c_{2}, \ldots, k_{2}$ nodes are controlled by the 2 nd player and similarly $a_{n}, b_{n}, c_{n}, \ldots, k_{n}$ nodes are controlled by the $n$th player.

In this network, the goal is maximizing players' payoff in the chain and determining the reliability of the entire chain by considering the uncertain amount of time, cost, and amount of flow by taking advantage of modeling in cooperative games in the nondeterministic circumstances. It should be noted that $a, b, c, \ldots, k$ are connecting nodes in the mentioned network. Let $\widetilde{N}=(V, A, \widetilde{\text { Cap }}, \widetilde{\text { Cost }}, \widetilde{T}, O, D)$ be a network with $O, D \in V$ being the source and the destination, respectively. In the network there are $n$ nodes which arrange a finite node set $V=\{1,2,3, \ldots, n\}$ and arc set of $A=\{(i, j) \mid i, j \in V\}$. In our terminology the accent “ $\sim$ " is used for emphasizing uncertain quantities. In the network, we consider that there are also three nonnegative stochastic random variables of $\widetilde{\text { Cap }}, \widetilde{\text { Cost }}$, and $\widetilde{T}$ defined as stochastic capacities, handling costs, and travel times for each arc in $A$, respectively.

3.2. Prerequisites and Assumptions. Prerequisites and assumptions are as follows:

(i) Each arc has a given and independent capacity in deterministic network, while it has different values in the scenario-based model.

(ii) Each arc has a given and independent transportation cost in deterministic network, while it has different values in the scenario-based model. 
(iii) Each arc has a given and independent travel time in deterministic network, while it has different values in the scenario-based model.

(iv) There is a budget limitation for each owner of the network.

(v) There is a time window to start from the origin and end at the destination, which must be observed by the network.

Notations. The indices, parameters, and decision variables are explained before providing the objective functions and proposing the model.

Indices are as follows:

$i$ : index of initial nodes.

$j$ : index of terminal nodes.

$k$ : index of nodes.

$O$ : the source node.

$D$ : the sink node.

$m$ : index of coalitions.

$c_{m}: m$ th coalition of the network owners.

Parameters are as follows:

Cap $_{i j}$ : capacity of arc between node $i$ and $j \in v$.

$T_{i j}$ : travel time between node $i$ and $j \in v$.

$T_{\min }$ : minimum allowed travel time in network $N$.

$T_{\max }:$ maximum allowed travel time in network $N$.

Cost $_{i j}$ : handing (shipping) cost per unit of flowing items between node $i$ and $j \in v$.

$B_{c_{m}}:$ the total budget available for coalition.

Decision variables are as follows:

$y_{i j}=\{1$, if arc between $i$ and $j$ is active; 0 , elsewhere $\}$, $\forall i, j \in V$.

$x_{i j}$ : flow between $i$ and $j \in v$.

$f_{c_{m}}$ : maximum-flow in the network controlled by coalition $m$.

Here the main goal in the network is maximizing players' payoff in the entire network. Moreover, determining the optimized reliability of the entire network with respect to the amount of transition time, cost, and capacity by using nondeterministic collaborative games models is considered. Therefore, this section presents mathematical linear programming based on triangular probability distribution for each possible coalition among players. Consider

$$
\text { Max } z=f_{c_{m}},
$$

Subject to: $\sum_{j} y_{O j}=1, \quad \forall j ; j=1,2, \ldots, n$,

$\sum_{i} y_{i D}=1, \quad \forall i ; i=1,2, \ldots, n$,

$\sum_{j} y_{i j} \leq 1, \quad \forall i, j \in v$,

$\sum_{i} y_{i j} \leq 1, \quad \forall i, j \in v$,

$\sum_{i} y_{i j}-\sum_{k} y_{j k}=0, \quad \forall i, j, k \in v$,

$x_{i j}-y_{i j} \cdot R_{i j}^{\mathrm{Cap}}\left(\mathrm{Cap}^{*}\right) \cdot \mathrm{Cap}^{*} \leq 0$,

$\forall i \neq j \in v$,

$$
\begin{aligned}
x_{\mathrm{O} j}-y_{\mathrm{O} j} \cdot R_{\mathrm{Oj}}^{\mathrm{Cap}}\left(\mathrm{Cap}^{*}\right) \cdot \mathrm{Cap}^{*} & \leq 0, \\
\forall j ; j & =1,2, \ldots, n,
\end{aligned}
$$

$x_{i D}-y_{i D} \cdot R_{i D}^{\mathrm{Cap}}\left(\mathrm{Cap}^{*}\right) \cdot \mathrm{Cap}^{*} \leq 0$,

$\forall i ; i=1,2, \ldots, n$,

$\sum_{i} x_{i j}-\sum_{k} x_{j k}=0, \quad \forall i \neq j \neq k \in v$,

$T_{\min }$

$$
\begin{gathered}
\leq \sum_{i, j} y_{i j} \cdot\left[1+\left(1-R_{i j}^{T}\left(T^{*}\right)\right)\right] \cdot T^{*}+\sum_{j} y_{\mathrm{O} j} \\
\cdot\left[1+\left(1-R_{O j}^{T}\left(T^{*}\right)\right)\right] \cdot T^{*}+\sum_{i} y_{i D} \\
\cdot\left[1+\left(1-R_{i D}^{T}\left(T^{*}\right)\right)\right] \cdot T^{*} \leq T_{\max }
\end{gathered}
$$

$$
\begin{aligned}
& \sum_{i, j} x_{i j} \cdot\left[1+\left(1-R_{i j}^{\text {Cost }}\left(\text { Cost }^{*}\right)\right)\right] \cdot \text { Cost }^{*} \\
& \quad+\sum_{j} x_{\mathrm{O} j} \cdot\left[1+\left(1-R_{\mathrm{Oj}}^{\text {Cost }}\left(\text { Cost }^{*}\right)\right)\right] \text { Cost }^{*} \\
& \quad+\sum_{i} x_{i D} \cdot\left[1+\left(1-R_{i D}^{\text {Cost }}\left(\text { Cost }^{*}\right)\right)\right] \cdot \text { Cost }^{*} \\
& \leq B_{c_{m}}
\end{aligned}
$$

$\forall i, j \in v$, 


$$
\begin{aligned}
& f_{c_{m}}-x_{i j}-M\left(1-y_{i j}\right) \leq 0, \quad \forall i, j \in v, \\
& y_{i j} \in\{0,1\}, \quad \forall i, j, \\
& x_{i j} \geq 0, \quad \forall i, j .
\end{aligned}
$$

In this model, capacity, cost, and travel time for each arc are independent random variables and are considered to deploy from a triangular density function with estimated parameters derived from optimistic, pessimistic, and most likely values. Therefore, the reliability terms in model could be calculated based on the triangular cumulative density function in exchange for target value for capacity, cost, and travel time which are constraints (16), (17), and (18). In these constraints, respectively, nominated value is presented by Cap ${ }^{*}$, Cost $^{*}$, and $T^{*}$. Consider

$$
\begin{aligned}
& R_{i j}^{\mathrm{Cap}}\left(\mathrm{Cap}^{*}\right)=\left\{\begin{array}{cl}
1-\frac{\left(\mathrm{Cap}^{*}-a_{\mathrm{Cap}_{i j}}\right)^{2}}{\left(b_{\mathrm{Cap}_{i j}}-a_{\mathrm{Cap}_{i j}}\right)\left(c_{\mathrm{Cap}_{i j}}-a_{\mathrm{Cap}_{i j}}\right)}, & \text { if: } a_{\mathrm{Cap}_{i j}} \leq \mathrm{Cap}^{*} \leq c_{\mathrm{Cap}_{i j}} \\
\frac{\left(b_{\mathrm{Cap}_{i j}}-\mathrm{Cap}^{*}\right)^{2}}{\left(b_{\mathrm{Cap}_{i j}}-a_{\mathrm{Cap}_{i j}}\right)\left(b_{\mathrm{Cap}_{i j}}-c_{\mathrm{Cap}_{i j}}\right)}, & \text { if: } c_{\mathrm{Cap}_{i j}} \leq \mathrm{Cap}^{*} \leq b_{\mathrm{Cap}_{i j}}
\end{array}\right\} \text {, }
\end{aligned}
$$

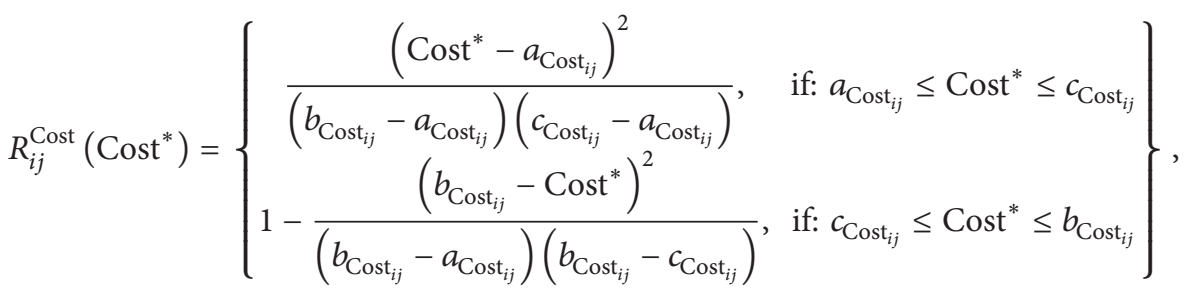

$$
\begin{aligned}
& R_{i j}^{T}\left(T^{*}\right)=\left\{\begin{array}{cl}
\frac{\left(T^{*}-a_{T_{i j}}\right)^{2}}{\left(b_{T_{i j}}-a_{T_{i j}}\right)\left(c_{T_{i j}}-a_{T_{i j}}\right)}, & \text { if: } a_{T_{i j}} \leq T^{*} \leq c_{T_{i j}} \\
1-\frac{\left(b_{T_{i j}}-T^{*}\right)^{2}}{\left(b_{T_{i j}}-a_{T_{i j}}\right)\left(b_{T_{i j}}-c_{T_{i j}}\right)}, & \text { if: } c_{T_{i j}} \leq T^{*} \leq b_{T_{i j}}
\end{array}\right\}
\end{aligned}
$$

Objective function (1) maximizes total flow of the network. Constraints (2)-(6) find the optimum path of the deterministic network. Constraints (7)-(9) are capacity-related constraints.

Constraint (10) is a balance constraint. Constraints (11) and (12) are in charge of observing time windows and budget limitations. Constraint (13) measures the flow of the network $f_{c_{m}}$. Constraints (14) and (15) are related to the variables of the problem. Constraints (16), (17), and (18) calculate reliability in continuous triangular distribution.

The purpose of optimization of the uncertain approach with continuous triangular distribution method is to optimize objective function at an acceptable level. Then, with solving the proposed model the value of the objective function is obtained. Furthermore, the reliability of the entire system and the value of the game in different coalitions are computed.

All ordinary linear programming packages such as Lingo or GAMS software can be used to solve this linear programming model. First, the basic idea is to solve this model by concerning the network of each owner independently. Then, the model of all coalitions of two owners must be solved. In the next step, all coalitions of three of the owners should be considered and this process continues until the grand coalition is achieved. Due to super additive characteristic of TU games, the utility of the network for any coalitional status must be greater than the sum of the utility of the network for the coalition's members; that is,

$$
f_{c_{m}}=v\left(c_{m}\right) \geq \sum_{p_{i} \subset c_{m}} v\left(p_{i}\right), \quad \forall c_{m} \in P .
$$

Actually, the difference between the sum of the "separate" maximum utility and the "mixed" maximum utility shows extra utility $\operatorname{EU}\left(c_{m}\right)$ created by the coalition $c_{m}$; that is,

$$
\mathrm{EU}\left(c_{m}\right)=v\left(c_{m}\right)-\sum_{p_{i} \subset c_{m}} v\left(p_{i}\right), \quad \forall c_{m} \in P
$$

According to the utility of the collaborating owners, the extra utility must be assessed. Therefore, the following criteria can be more reliable measure for the synergy of a coalition:

$$
\operatorname{Synergy}\left(c_{m}\right)=\frac{\operatorname{EU}\left(c_{m}\right)}{v\left(c_{m}\right)} .
$$

\section{Results and Discussion}

4.1. Illustrative Example. To verify and authenticate the validity of the proposed model and to prove its functionality, 
TABLE 1: Capacities, travel times, and transportation costs.

\begin{tabular}{|c|c|c|c|c|c|c|c|c|c|}
\hline \multirow{2}{*}{ Pair } & \multicolumn{3}{|c|}{ Cap $\left(\mathrm{m}^{3}\right)$} & \multicolumn{3}{|c|}{$T(\mathrm{~h})$} & \multicolumn{3}{|c|}{ Cost (dollars) } \\
\hline & $a$ & $c$ & $b$ & $a$ & $c$ & $b$ & $a$ & $c$ & $b$ \\
\hline$(1-2)$ & 540 & 681 & 892 & 33 & 82 & 124 & 88 & 106 & 139 \\
\hline$(1-3)$ & 596 & 842 & 886 & 40 & 68 & 130 & 84 & 93 & 126 \\
\hline$(1-4)$ & 533 & 784 & 976 & 30 & 97 & 130 & 70 & 109 & 128 \\
\hline$(2-3)$ & 638 & 651 & 919 & 33 & 78 & 133 & 76 & 114 & 130 \\
\hline$(2-7)$ & 505 & 686 & 958 & 34 & 60 & 120 & 82 & 96 & 135 \\
\hline$(3-5)$ & 536 & 834 & 891 & 43 & 62 & 119 & 71 & 108 & 134 \\
\hline$(3-6)$ & 582 & 736 & 947 & 40 & 91 & 129 & 89 & 97 & 135 \\
\hline$(4-6)$ & 535 & 674 & 941 & 33 & 78 & 130 & 86 & 101 & 134 \\
\hline$(4-7)$ & 562 & 819 & 975 & 27 & 87 & 127 & 81 & 117 & 126 \\
\hline$(5-8)$ & 548 & 674 & 991 & 40 & 79 & 129 & 81 & 110 & 131 \\
\hline$(6-8)$ & 608 & 755 & 999 & 26 & 57 & 114 & 71 & 103 & 129 \\
\hline$(6-9)$ & 555 & 803 & 944 & 43 & 106 & 139 & 74 & 95 & 134 \\
\hline$(7-6)$ & 514 & 756 & 930 & 46 & 80 & 122 & 84 & 113 & 131 \\
\hline$(7-9)$ & 552 & 680 & 938 & 28 & 53 & 132 & 75 & 104 & 134 \\
\hline$(8-9)$ & 554 & 798 & 909 & 41 & 93 & 123 & 70 & 100 & 129 \\
\hline
\end{tabular}

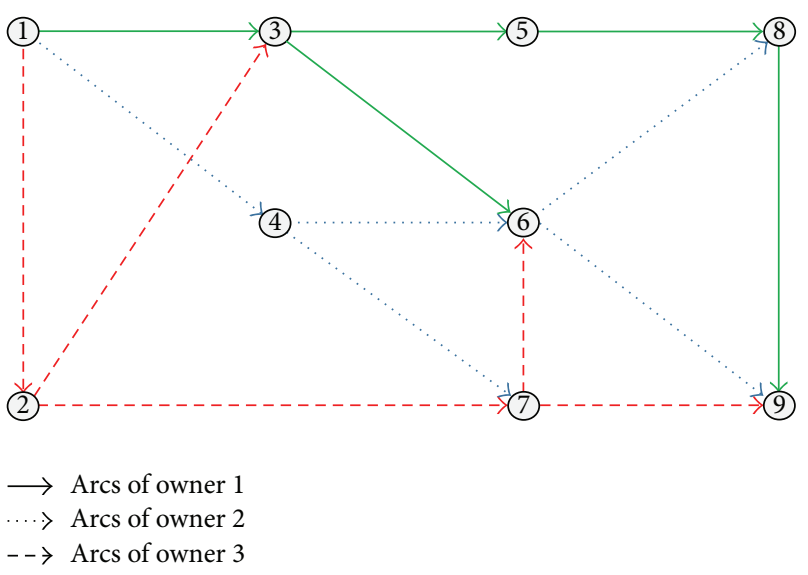

FIGURE 2: Assumed network and links [17].

a case study is used. For this purpose, a transmission network with three competitive suppliers is considered that is shown in Figure 2. The first supplier (or the first player) is controlling the set $\{(1,3),(3,5),(3,6),(5,8),(8,9)\}$, the second one is controlling the set $\{(1,4),(4,6),(4,7),(6,8),(6,9)\}$, and the third supplier is controlling the set $\{(1,2),(2,3),(2,7)$, $(7,6),(7,9)\}$. It should be noted that it is assumed that the data have normal distribution. The goal of the problem is to maximize flow from the original node to the destination node.

In Table 1, the amount of transmission capacity, time of transition and distribution between the nodes, and transmission costs in pessimistic cases (a), most likely cases (b), and the most optimistic cases (c) are presented.

To solve the problem, $T_{\min }$ and $T_{\max }$ are considered to be $100 \mathrm{~h}$ and $500 \mathrm{~h}$, respectively. Moreover, the available budget for the first player is $5000 \$$, the available budget for the second player is $10,000 \$$, and the available budget for the third player is $15,000 \$$. After solving the model by GAMS
TABLE 2: The values of reliability.

\begin{tabular}{cccc}
\hline Arcs & $R$ (cap) & $R(T)$ & $R$ (cost) \\
\hline$(1-2)$ & 0.794 & 0.974 & 0.848 \\
$(1-3)$ & 0.972 & 0.954 & 0.994 \\
$(1-4)$ & 0.895 & 0.922 & 0.977 \\
$(2-3)$ & 0.998 & 0.934 & 0.943 \\
$(2-7)$ & 0.774 & 0.993 & 0.930 \\
$(3-5)$ & 0.896 & 0.994 & 0.926 \\
$(3-6)$ & 0.938 & 0.933 & 0.918 \\
$(4-6)$ & 0.801 & 0.949 & 0.924 \\
$(4-7)$ & 0.941 & 0.958 & 0.978 \\
$(5-8)$ & 0.845 & 0.949 & 0.939 \\
$(6-8)$ & 0.981 & 1.000 & 0.976 \\
$(6-9)$ & 0.923 & 0.803 & 0.948 \\
$(7-6)$ & 0.840 & 0.980 & 0.924 \\
$(7-9)$ & 0.840 & 0.961 & 0.932 \\
$(8-9)$ & 0.913 & 0.967 & 0.979 \\
\hline
\end{tabular}

TABLE 3: Characteristic function and optimal flows for risk behavior of coalition's member under different aggregation methods.

\begin{tabular}{lccc}
\hline Coalition & $f_{c_{m}}=V\left(c_{m}\right)$ & $\mathrm{EU}\left(c_{m}\right)$ & Synergy $\left(c_{m}\right)$ \\
\hline$C\{1\}$ & 27 & 0 & 0 \\
$C\{2\}$ & 76 & 0 & 0 \\
$C\{3\}$ & 119 & 0 & 0 \\
$C\{1,2\}$ & 114 & 11 & 0.096 \\
$C\{1,3\}$ & 159 & 13 & 0.081 \\
$C\{2,3\}$ & 199 & 4 & 0.02 \\
$C\{1,2,3\}$ & 253 & 31 & 0.122 \\
\hline
\end{tabular}

software, the results of reliability of links are shown in Table 2. Table 3 indicates the final output of the proposed model. 
It should be mentioned that, in the network contract, optimal transmission capacity, time, and cost are considered as follows: Cap $^{*}=641, T^{*}=114$, and Cost ${ }^{*}=123$. For example, consider a part of a pipeline has a random capacity over time within the range of [540-892] cubic meters. Experts believed that most of the time this part conducted $681 \mathrm{~m}^{3}$. Here as a common sense we could consider that this part of pipeline has a triangular random variable and according to constraint (16), it has the chance of $79.4 \%$ to conduct $641 \mathrm{~m}^{3}$.

4.2. Graph Collaborative Arrangement for the Multiple-Owner Graph. When the utility of coalition of graph owners is computed, the problem of sharing the benefits of the collaboration among variant owners should be considered. The problem is difficult to solve because the contribution of each owner to the utility of graph is ambiguous. Therefore, a theoretically grounded technique is needed and CGT can be the best option. In the first place, some basic concepts related to CGT are considered and then they are developed for multipleowner graph in nondeterministic circumstances.

For each player, the set $P$ is $P=\{1,2, \ldots, N\}$, and $v(P)$ shows the available payoff when all players cooperate. Now, consider $y_{i}$ is a real number for each player $i=1,2, \ldots, N$, with $\sum_{i=1}^{N} y_{i} \leq v(P)$. If a vector $\vec{y}=\left(y_{1}, y_{2}, \ldots, y_{n}\right)$ is suitable for individual and group rationality conditions, that is, $y_{i} \geq$ $v(i)$ for all $i \in P$ and $\sum_{i=1}^{N} y_{i}=v(P)$, then it will be considered as an imputation.

Imputation is an allocation or a payoff vector and it means how $v(P)$ is to be distributed to players so that no player rejects the allocated payoff. The set of all imputations for the competitive game is $Y=\left\{\vec{y}=\left(y_{1}, y_{2}, \ldots, y_{n}\right) \mid y_{i} \geq\right.$ $\left.v(i), \sum_{i=1}^{N} y_{i}=v(P)\right\}$. Specifying the imputation form $Y$, which yields a fair allocation of total payoff, is the main goal in CGT. Several assignment methods based on different interpretation of fair allocation are developed that some of them are reviewed briefly $[32,33]$.

The excess of coalition $C \in P$ for imputation $\vec{y} \in Y$ is equal to $e(C, y)=v(C)-\sum_{i \in C} y_{i}$. It indicates that the amount of payoff allocated to the coalition $C$ varies from the payoff associated with $C$. The core of the game can be defined as follows:

$$
\begin{aligned}
\text { core }(0) & =\{\vec{y} \in Y \mid e(C, \vec{y}) \leq 0, \forall C \subset P\} \\
& =\left\{\vec{y} \in Y \mid v(C) \leq \sum_{i=1}^{N} y_{i}, \forall C \subset P\right\} .
\end{aligned}
$$

As a matter of fact, the core is the set of all imputations so that each coalition achieves least payoff associated with the corresponding coalition. The game is called stable if the core is nonempty. Besides, for the real number $\varepsilon, \operatorname{core}(\varepsilon)$ can be defined as follows:

$$
\begin{aligned}
\operatorname{core}(\varepsilon) \\
=\{\vec{y} \in Y \mid e(C, \vec{y}) \leq \varepsilon, \forall C \subset P, C \neq P, C \neq \varnothing\} .
\end{aligned}
$$

TABLE 4: Assigning of the coalition payoff by variant methods.

\begin{tabular}{ccccc}
\hline & Owner & Shapley & $\tau$-value & Core center \\
\hline \multirow{4}{*}{ Coalition } & $\{1\}$ & 40 & 39.877 & 39.673 \\
& $\{2\}$ & 84.5 & 84.585 & 84.688 \\
& $\{3\}$ & 128.5 & 128.538 & 128.638 \\
& Stable & Yes & Yes & Yes \\
\hline
\end{tabular}

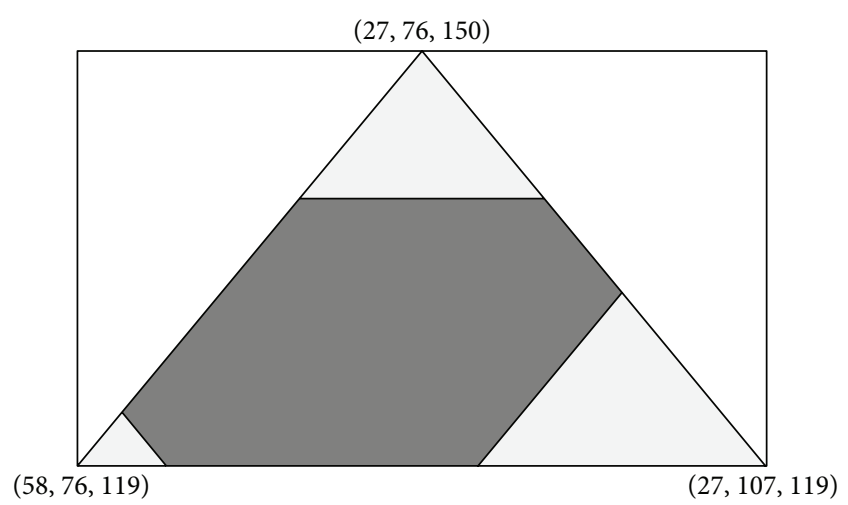

Figure 3: Core for the multiple-owner graph example under variant risk attitudes.

An assignment method based on four axioms of efficiency, symmetry, additive, and dummy property has been formulated by Shapley in [34]. An imputation $\vec{y}=\left(y_{1}, y_{2}, \ldots, y_{n}\right)$ demonstrates Shapley value if

$$
\begin{array}{r}
y_{i}=\sum_{C \subset \Pi^{i}}[v(C)-v(C-\{i\})] \frac{(|C|-1) !(N-|C|) !}{N !}, \\
i=1,2, \ldots, n,
\end{array}
$$

where $\Pi^{i}$ is the set of all coalitions $C \subset P$ submitting player $i$ as a member and $|C|$ shows number of members in $C$.

The imputations obtained by different TU game approaches including the Shapley value, the $\tau$-value, and the core center are presented in Table 4.

Figure 3 indicates that the imputation sets and corresponding cores in barycentric coordinates are calculated by TUGlab package [35].

The difference between allocated utility obtained from grand coalition and utility if the coalition $C$ performs independently is satisfaction of a coalition $C$ from imputation $\vec{y}=\left(y_{1}, y_{2}, \ldots, y_{n}\right)$; that is, $F_{s}(C, \vec{y})=\sum_{i \in C} y_{i}-v(C)=$ $-e(C, y)$. Table 4 indicates the corresponding satisfaction values $F_{s}(C, \vec{y})$, as well as relative values $F_{s}(C, \vec{y}) / v(C)$ for imputations of Table 3. This table shows that when the size of the coalition increases absolute and relative satisfactions of coalition will reduce. It shows that when a coalition gets larger, the obtained advantages of adding new members will decline. In real situations, the complexity involved in collaboration of multiple owners makes reaching these advantages difficult.

Moreover, Table 5 indicates the coalition satisfactions in situation. Thus, in this example, when the coalitions have a 
TABLE 5: Coalition satisfactions for variant TU game approaches.

\begin{tabular}{ccccc}
\hline & Coalition & Shapley & $\tau$-value & Core center \\
\hline & $C_{1}=\{1\}$ & 13 & 12.877 & 12.673 \\
& $(48.1 \%)$ & $(47.7 \%)$ & $(46.9 \%)$ \\
& $C_{2}=\{2\}$ & 7.5 & 8.585 & 8.688 \\
& $(10 \%)$ & $(11.3 \%)$ & $(11.4 \%)$ \\
& & 9.5 & 9.538 & 9.638 \\
& $C_{3}=\{3\}$ & $(8 \%)$ & $(8 \%)$ & $(8.1 \%)$ \\
& & 10.5 & 10.462 & 10.361 \\
& $C_{4}=\{1,2\}$ & $(9.2 \%)$ & $(9.1 \%)$ & $(9.1 \%)$ \\
& & 9.5 & 9.415 & 9.311 \\
& $C_{5}=\{1,3\}$ & $(6 \%)$ & $(5.95 \%)$ & $(5.8 \%)$ \\
& & 14 & 14.123 & 14.326 \\
& $C_{6}=\{2,3\}$ & $(7 \%)$ & $(7.1 \%)$ & $(7.2 \%)$ \\
\hline
\end{tabular}

high risk adverse attitude, the owners show less interest to participate in the coalition.

Table 5 illustrates the correlation measure for each pair of CGT methods. The solution of different CGT methods leads to different solutions. The following observation and managerial insights are derived from the numerical examples:

(1) With regard to cooperation between the players, the synergy between the players is remarkable. For example, the synergy between the players in multilateral coalition is 0.122 , which can serve as a good incentive for cooperation between them in terms of the network.

(2) Table 3 indicates that combining cooperation between the players leads to an increase in the reliability of the system. Therefore, the value of the game has been increased.

(3) Table 5, according to the consent of the coalition in the network, indicates that player's considerations regarding network reliability and uncertainty tend to have a coalition together.

\section{Conclusions}

In traditional network games, rottenly analysis is performed under deterministic conditions. Thus, estimates may be noisy due to lack of real attention to possible risky events. Our optimization method proposed a novel approach to overcome risky conditions on the maximum-flow problems on cooperative circumstances. This method covers more reliability in decision-making under uncertain conditions and acts as a valid solution under full certainty. Another advantage of the proposed method is simplicity in using a well-known triangular probability distribution. Such methods help decision makers to benefit from maximum experts' viewpoints and there is no need to follow statistical distributions fitting method based on historical data. The method needs to have optimistic, pessimistic, and most likely estimates for any risk factors. The results of numerical example indicate that the mathematical model is efficient for cooperation among players and increases reliability.
There are some directions and suggestions for future research works. Researches may consider more risky factors, inventory management disciplines, and competition in the amount of sending flow to retailers. Moreover, it seems that applying our proposed method could satisfy the need for building the cooperative strategies for stochastic networks managed by multiple owners/players under uncertain time, cost, and capacity parameters of the network's arcs. Last but not least, fuzzy-based methods can be employed in order to increase network reliability in further research.

\section{Competing Interests}

The authors declare that they have no competing interests.

\section{References}

[1] R. Lancioni, H. J. Schau, and M. F. Smith, "Internet impacts on supply chain management," Industrial Marketing Management, vol. 32, no. 3, pp. 173-175, 2003.

[2] K. A. Patterson, C. M. Grimm, and T. M. Corsi, "Adopting new technologies for supply chain management," Transportation Research Part E: Logistics and Transportation Review, vol. 39, no. 2, pp. 95-121, 2003.

[3] J. Timmer, M. Chessa, and R. J. Boucherie, "Cooperation and game-theoretic cost allocation in stochastic inventory models with continuous review," European Journal of Operational Research, vol. 231, no. 3, pp. 567-576, 2013.

[4] A. Schrijver, "On the history of the transportation and maximum flow problems," Mathematical Programming, vol. 91, no. 3, pp. 437-445, 2002.

[5] M. Frisk, M. Göthe-Lundgren, K. Jörnsten, and M. Rönnqvist, "Cost allocation in collaborative forest transportation," European Journal of Operational Research, vol. 205, no. 2, pp. 448458, 2010.

[6] N. Lehoux, J.-F. Audy, S. D’amours, and M. Rönnqvist, "Issues and experiences in logistics collaboration," IFIP Advances in Information and Communication Technology, vol. 307, pp. 6976, 2009.

[7] S. Lozano, P. Moreno, B. Adenso-Díaz, and E. Algaba, "Cooperative game theory approach to allocating benefits of horizontal cooperation," European Journal of Operational Research, vol. 229, no. 2, pp. 444-452, 2013.

[8] C. Vanovermeire and K. Sörensen, "Measuring and rewarding flexibility in collaborative distribution, including two-partner coalitions," European Journal of Operational Research, vol. 239, no. 1, pp. 157-165, 2014.

[9] S. Saha, S. Panda, N. M. Modak, and M. Basu, "Mail-in-rebate coupled with revenue sharing and downward direct discount for supply chain coordination," International Journal of Operational Research, vol. 23, no. 4, pp. 451-476, 2015.

[10] E. Kalai and E. Zemel, "Generalized network problem yielding totally balanced games," Operation Research, vol. 30, no. 5, pp. 998-1008, 1982.

[11] S. L. Charles and D. R. Hansen, "An evaluation of activitybased costing and functional-based costing: a game-theoretic approach," International Journal of Production Economics, vol. 113, no. 1, pp. 282-296, 2008.

[12] S. Zibaei, A. Hafezalkotob, and S. S. Ghashami, "Cooperative vehicle routing problem: an opportunity for cost saving," 
Journal of Industrial Engineering International, vol. 12, no. 3, pp. 271-286, 2016.

[13] R. Zhao, G. Neighbour, J. Han, M. McGuire, and P. Deutz, "Using game theory to describe strategy selection for environmental risk and carbon emissions reduction in the green supply chain," Journal of Loss Prevention in the Process Industries, vol. 25, no. 6, pp. 927-936, 2012.

[14] M. G. H. Bell, "Measuring network reliability: a game theoretic approach," Journal of Advanced Transportation, vol. 33, no. 2, pp. 135-146, 1999.

[15] P. M. Reyes, "Logistics networks: a game theory application for solving the transshipment problem," Applied Mathematics and Computation, vol. 168, no. 2, pp. 1419-1431, 2005.

[16] J. R. San Cristóba, "Cost allocation between activities that have caused delays in a project using game theory," Procedia Technology, vol. 16, pp. 1017-1026, 2014.

[17] A. Hafezalkotob and A. Makui, "Cooperative maximum-flow problem under uncertainty in logistic networks," Applied Mathematics and Computation, vol. 250, pp. 593-604, 2015.

[18] R. A. McCain, "Cooperative games and cooperative organizations," The Journal of Socio-Economics, vol. 37, no. 6, pp. 21552167, 2008.

[19] M. Esmaeili, N. S. Gamchi, and E. Asgharizadeh, "Threelevel warranty service contract among manufacturer, agent and customer: a game-theoretical approach," European Journal of Operational Research, vol. 239, no. 1, pp. 177-186, 2014.

[20] L. Wu, Q. Tan, and Y. Zhang, "Delivery time reliability model of logistics network," Mathematical Problems in Engineering, vol. 2013, Article ID 879472, 5 pages, 2013.

[21] W. Y. Szeto, "Cooperative game approaches to measuring network reliability considering paradoxes," Transportation Research Part C: Emerging Technologies, vol. 19, no. 2, pp. 229241, 2011.

[22] X. Zhao, D. Wang, Y. Yan, and Z. Gu, "Stochastic simulation on system reliability and component probabilistic importance of road network," Mathematical Problems in Engineering, vol. 2015, Article ID 456485, 5 pages, 2015.

[23] S. A. Hosseini and E. Wadbro, "Connectivity reliability in uncertain networks with stability analysis," Expert Systems with Applications, vol. 57, pp. 337-344, 2016.

[24] A. Amin, L. Grunske, and A. Colman, "An approach to software reliability prediction based on time series modeling," Journal of Systems and Software, vol. 86, no. 7, pp. 1923-1932, 2013.

[25] W. E. Kuo, Optimal Reliability Design: Fundamentals and Applications, Cambridge University Press, New York, NY, USA, 2001.

[26] W.-C. Yeh, C. Bae, and C.-L. Huang, "A new cut-based algorithm for the multi-state flow network reliability problem," Reliability Engineering \& System Safety, vol. 136, pp. 1-7, 2015.

[27] K. Khalili-Damghani, A.-R. Abtahi, and M. Tavana, "A new multi-objective particle swarm optimization method for solving reliability redundancy allocation problems," Reliability Engineering \& System Safety, vol. 111, pp. 58-75, 2013.

[28] K. Hausken, "Probabilistic risk analysis and game theory," Risk Analysis, vol. 22, no. 1, pp. 17-27, 2002.

[29] R. S. Prabhu Gaonkar, M. Xie, and X. Fu, "Reliability estimation of maritime transportation: a study of two fuzzy reliability models," Ocean Engineering, vol. 72, pp. 1-10, 2013.

[30] Y. Jiang, H.-J. Sun, and J.-J. Wu, "Comparative analysis of transportation network design problem under stochastic capacity," Journal of Transportation Systems Engineering and Information Technology, vol. 14, no. 3, pp. 85-90, 2014.
[31] X. Zhang, E. Miller-Hooks, and K. Denny, "Assessing the role of network topology in transportation network resilience," Journal of Transport Geography, vol. 46, pp. 35-45, 2015.

[32] Y. F. Huang, W. L. Ye, and F. F. Zhou, "Research on the profit distribution of logistics company strategic alliance based on shapley value," Advanced Materials Research, vol. 765-767, pp. 3253-3257, 2013.

[33] H. S. Fathabadi and M. Ghiyasvand, "A new algorithm for solving the feasibility problem of a network flow," Applied Mathematics and Computation, vol. 192, no. 2, pp. 429-438, 2007.

[34] R. Myerson, Game Theory: Analysis of Conflict, Harvard University, Cambridge, Mass, USA, 1991.

[35] M. Calvo, M. Angel, S. Rodríguez, and Estela, TUGlab: A Cooperative Game Theory Toolbox, 2006. 


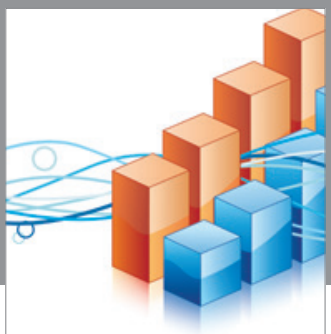

Advances in

Operations Research

vatem alat4

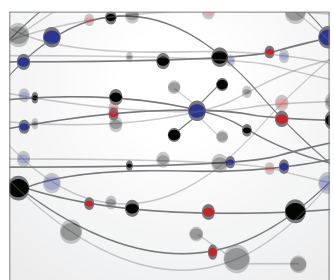

\section{The Scientific} World Journal
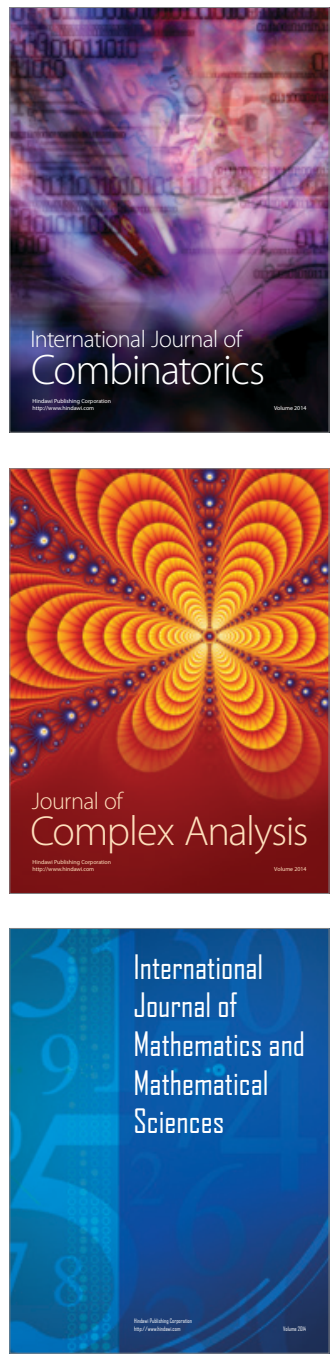
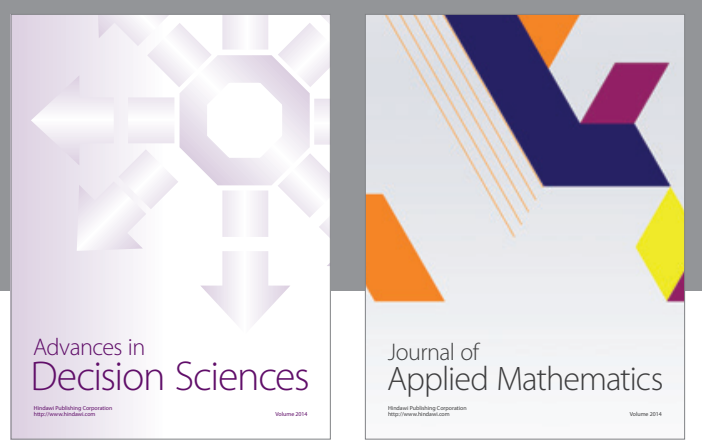

Algebra

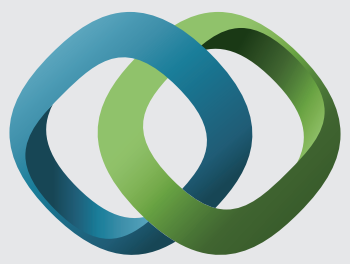

\section{Hindawi}

Submit your manuscripts at

http://www.hindawi.com
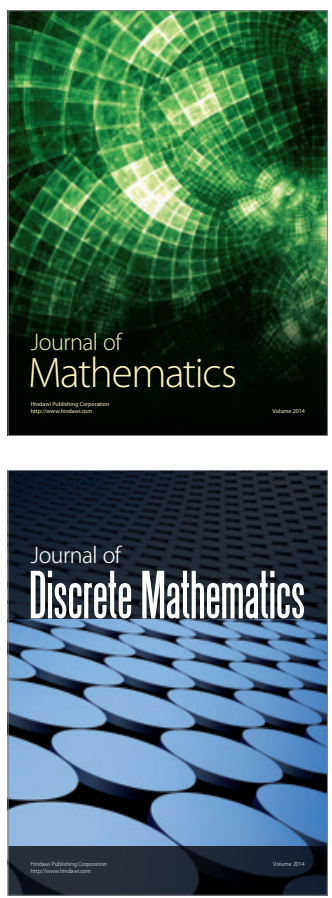

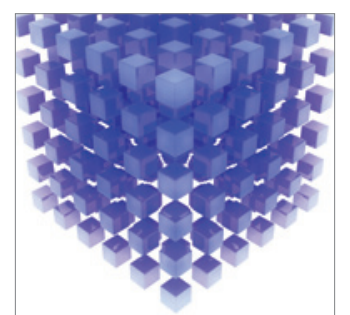

Mathematical Problems in Engineering
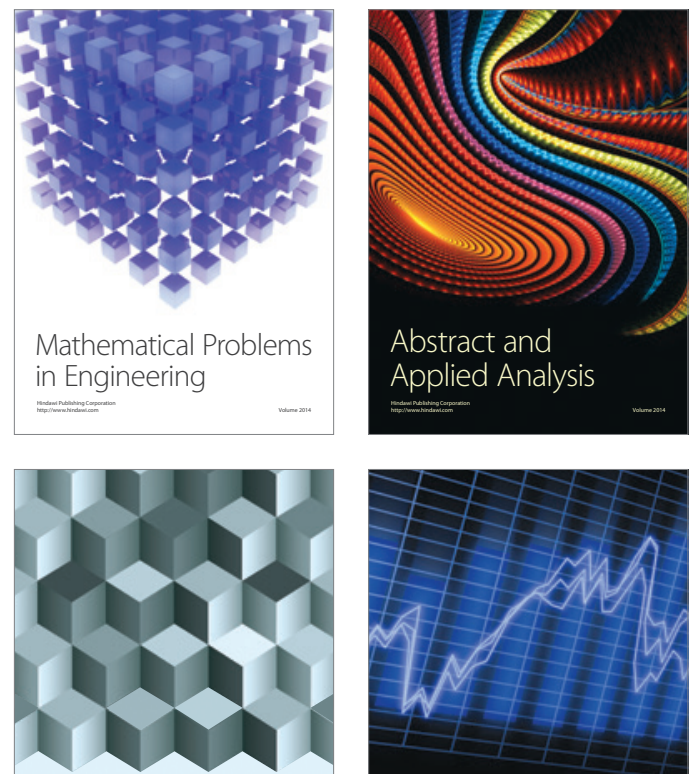

Journal of

Function Spaces

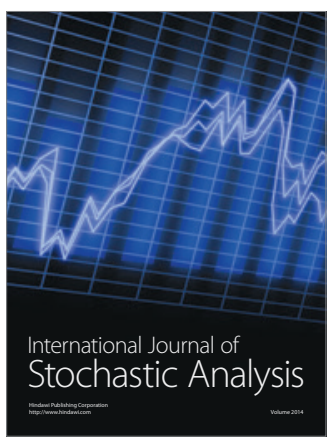

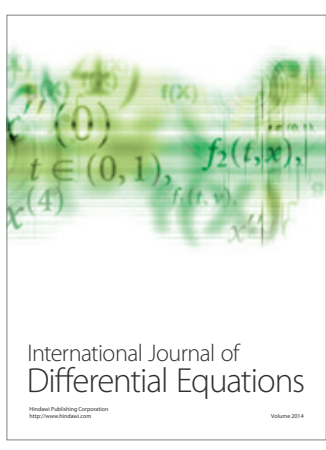
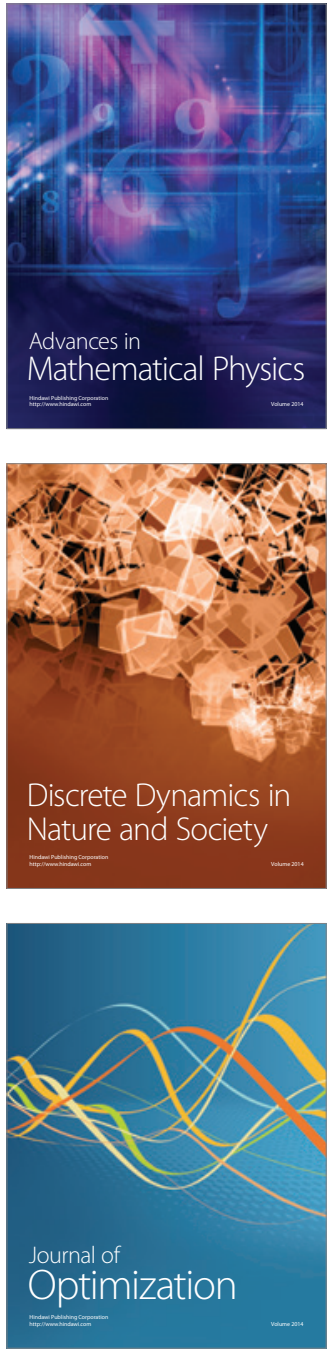